\title{
Lipopolysaccharide Masking of Gonococcal Outer-membrane Proteins Modulates Binding of Bactericidal Cathepsin G to Gonococci
}

\author{
By WILLIAM M. SHAFER \\ Department of Microbiology and Immunology, Emory University School of Medicine, \\ and The Veterans Administration, Atlanta, Ga. 30322, USA
}

(Received 22 June 1987; revised 13 October 1987)

\begin{abstract}
Human polymorphonuclear leucocyte (PMN) lysosomal cathepsin $\mathrm{G}$ exerts potent bactericidal action against Neisseria gonorrhoeae in vitro, independent of its serine esterase activity. The results presented demonstrate that (1) bactericidal, diisopropylfluorophosphate-treated cathepsin $G$ binds in a specific and saturable manner to the surface of gonococci, (2) loss of carbohydrates in gonococcal LPS due to mutation increases total and specific binding of cathepsin G, and (3) at least three outer-membrane proteins (OMPs) (PIA, PIII, and a $45 \mathrm{kDa}$ OMP) interact with cathepsin G. Taken together, the results suggest that gonococcal susceptibility to the lethal action of cathepsin G, and perhaps susceptibility of gonococci to oxygen-independent killing by PMNs, is controlled by LPS-masking of cathepsin-G-binding OMPs.
\end{abstract}

\section{INTRODUCTION}

Human PMN lysosomal cathepsin G exerts potent bactericidal activity in vitro against Neisseria gonorrhoeae (Shafer et al., 1986a, b). Hence, cathepsin G is a candidate for nonoxidative killing of gonococci by PMNs. This is important since previous studies (Casey $e t$ al., 1986; Rest et al., 1982) have demonstrated the capacity of PMNs to kill gonococci by nonoxidative means, in the absence of oxidative antimicrobial systems.

The antigonococcal action of cathepsin $\mathrm{G}$ is independent of its serine esterase activity (Shafer et $a l ., 1986 a, b)$ and is probably due to its highly cationic nature (Odeberg \& Olsson, 1975). Recent studies described a spontaneous LPS mutant of gonococci that exhibited hypersusceptibility to the lethal action of cathepsin G (Shafer et al., 1986a). Using this mutant and its more cathepsin-G-resistant parental strain it was found that certain OMPs were candidates for binding cathepsin $G$ since they were susceptible to cleavage by enzymically active cathepsin $G$ (Shafer \& Morse, 1987). Moreover, surface-exposure and susceptibility of OMPs PIII and PIA appeared to be greater in the OM of the LPS mutant. This suggested that the mutation affecting LPS structure augmented exposure of these OMPs due to loss of carbohydrates that normally mask them.

Enhanced susceptibility of OMPs in the LPS mutant to proteolytic cleavage by cathepsin G suggested that their greater surface exposure might promote binding of cathepsin G. To test this hypothesis, the binding of radiolabelled cathepsin $\mathrm{G}$ to the surface of isogenic strains differing in susceptibility to the lethal action of cathepsin $G$ was evaluated.

\section{METHODS}

Preparation and iodination of cathepsin G. Human PMN lysosomal cathepsin G was prepared from acetate extracts (Rest et al., 1977) of isolated granules as described by Shafer $e t$ al. (1986b). The granulocyte donors denied previous history of gonococcal infection and were not under antibiotic treatment. Using Iodogen (Pierce), $100 \mu \mathrm{g}$

Abbreviations: DFP, diisopropylfluorophosphate; OMP, outer-membrane protein; PMN, polymorphonuclear leucocyte. 
purified cathepsin G, determined by the method of Bradford (1976), was iodinated as described by Farley $e t$ al . (1987). After iodination, samples were treated with DFP (Shafer et al., 1984) and dialysed at $4{ }^{\circ} \mathrm{C}$ against several changes of PBS $(0.145 \mathrm{M}$-sodium chloride, $0.145 \mathrm{M}$-sodium phosphate, $\mathrm{pH} 7.4)$. The specific activity of the radioiodinated cathepsin G (with or without DFP treatment) was in the range $1-5 \times 10^{5} \mathrm{c} . \mathrm{p} . \mathrm{m}$. per $\mu \mathrm{g}$ cathepsin G. Radioiodinated preparations of cathepsin $G$ were examined by SDS-PAGE (Laemmli, 1970) under reducing conditions. The slab gel was stained with silver (Shafer et al., 1984), dried onto Whatman filter paper and applied to Kodak X-OMAT AR-5 X-ray film for autoradiography. The results (Fig. 1) showed that the DFP-treated cathepsin G migrated as a 24-25.5 kDa triplet (lane A), characteristic of the known isoenzymes (Travis et al., 1980), while the enzymically active preparation revealed the presence of minute quantities of additional polypeptides of molecular mass $<14 \mathrm{kDa}$ (lane $\mathrm{B}$ ).

Strains of $N$. gonorrhoeae. Strains FA102 and WS1 were used throughout this study. Strain WS1, a spontaneous pyocin-resistant mutant of FA102, produces a truncated LPS (Shafer et al., 1986a) but has a similar OMP profile to parental strain FA102 (Shafer \& Morse, 1987); both strains produce PIA, serovar PIA1. WS1 is at least tenfold more susceptible than FA102 to the lethal action of enzymically active and inactive preparations of cathepsin G (Shafer et al., 1986a, b). For routine culturing, both strains were passed on GCB agar containing defined supplements (Shafer et al., 1986a) as OP-P- variants.

Analysis of the binding of cathepsin $G$ to gonococci. Gonococci were harvested from GCB agar plates and grown in GCB broth containing supplements and $0.043 \%(w / v)$ sodium bicarbonate. All cultures were grown at $37^{\circ} \mathrm{C}$ with shaking to an $\mathrm{OD}_{550}$ of $\mathbf{0} \cdot 35$. Samples $(1 \mathrm{ml})$ were added to Eppendorf microfuge tubes containing iodinated cathepsin G, and after brief vortexing, the mixtures were incubated at the indicated temperature. At indicated intervals, samples were removed and the bacteria were collected by centrifugation for $2 \mathrm{~min}$ at $13500 \mathrm{~g}$. The supernatants were removed and the pellets analysed for bound ${ }^{125} \mathrm{I}$ using a Beckman LS 4000 gamma scintillation counter. Each experiment was done in triplicate. In the absence of added gonococci, about $10 \%$ of the labelled cathepsin $\mathrm{G}$ bound to the sides of the microfuge tubes when incubations were done in GCB broth alone under the same conditions. Binding was assessed as $\mu \mathrm{g}$ of cathepsin $\mathrm{G}$ bound per $10^{7}$ c.f.u. added. In several experiments determination of the total number of diplococcal particles by Coulter counter $(20 \mu \mathrm{m}$ aperture $)$ measurements showed that about $90 \%$ of the added gonococci (FA102 and WS1) were viable (viability was determined by plating on GCB agar).

Immunoprecipitation of gonococcal OMPs bound to cathepsin $G$. Surface-iodinated gonococci were obtained as described by Judd (1982). After washing with PBS, the labelled gonococci were added to microfuge tubes containing unlabelled cathepsin $\mathrm{G}\left(100 \mu \mathrm{g} \mathrm{ml}^{-1}\right)$ or an equivalent volume of PBS. The mixtures were incubated at $22{ }^{\circ} \mathrm{C}$ for $30 \mathrm{~min}$, centrifuged, washed twice with PBS, and the pellet was resuspended in $100 \mu 11 \%(\mathrm{w} / \mathrm{v}) \mathrm{SB}-12$ (Calbiochem, Behring Diagnostics) and incubated at $37^{\circ} \mathrm{C}$ for $30 \mathrm{~min}$. The SB-12 extracts were collected by centrifugation as described by Joiner et al. (1983), then treated with $500 \mu \mathrm{g}$ IgG purified from a goat anti-cathepsin $\mathrm{G}$ antiserum (kindly provided by James Travis, University of Georgia, Athens, Ga.). After overnight incubation at $4{ }^{\circ} \mathrm{C}$, the mixtures were treated with $25 \mu \mathrm{l}$ rabbit anti-goat IgG antiserum (heavy and light chain specific; Cappel $\mathrm{Labs}$ ) and incubated for $1 \mathrm{~h}$ at $37^{\circ} \mathrm{C}$. Immunoprecipitates were collected by centrifugation, treated with SDS-PAGE solubilizing buffer, boiled for $5 \mathrm{~min}$ and subjected to electrophoresis (Laemmli, 1970). After electrophoresis, the gel was stained with Coomassie Brilliant Blue, destained with $10 \%(\mathrm{v} / \mathrm{v})$ acetic acid containing $1 \%(\mathrm{v} / \mathrm{v})$ glycerol and dried onto filter paper. The dried gel was exposed to X-ray film at $-80^{\circ} \mathrm{C}$ for $24 \mathrm{~h}$, the film developed, and the dried gel exposed to fresh X-ray film for an additional $96 \mathrm{~h}$.

\section{RESULTS}

Earlier studies by Shafer \& Morse (1987) showed that enzymically active cathepsin G cleaved gonococcal OMPs in situ while DFP-treated cathepsin G did not. Since both preparations of cathepsin $\mathrm{G}$ exerted similar bactericidal action (Shafer et al., 1986a, b), experiments were designed to test whether DFP-treated cathepsin G binds to the surface of gonococci in a specific and saturable manner, whether loss of carbohydrates in LPS enhances binding and if surfaceexposed OMPs interact with cathepsin G.

\section{Binding of DFP-treated cathepsin G to strain FA102}

Binding of DFP-treated cathepsin G, labelled with ${ }^{125}$ I (Fig. 1, lane A), was first evaluated using strain FA102 in the standard binding assay described in Methods. The binding assay was done at 4,22 and $37^{\circ} \mathrm{C}$ with samples removed at various times. The results (not presented) showed that binding was rapid (complete within $1 \mathrm{~min}$ ) and relatively independent of temperature. Approximately $18 \%$ of the added $(1 \mu \mathrm{g})$ cathepsin $\mathrm{G}$ was bound to the gonococcal pellet. 


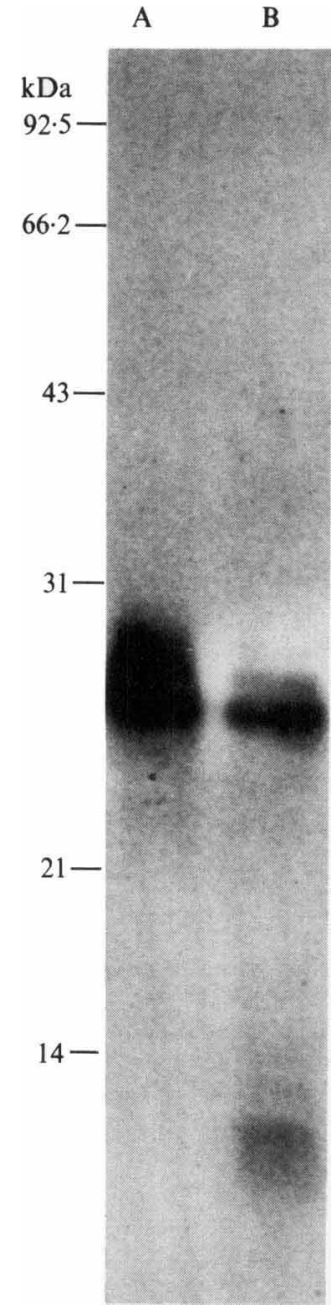

Fig. 1

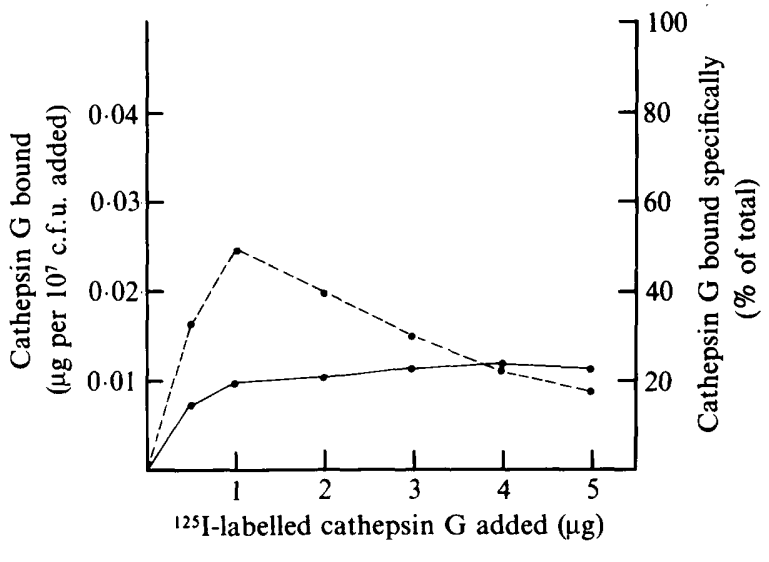

Fig. 2

Fig. 1. Autoradiographic profile of ${ }^{125} \mathrm{I}$-labelled cathepsin $\mathrm{G}$ treated or not treated with DFP. Lane A, DFP-treated cathepsin G; lane B, untreated cathepsin G showing low-molecular-mass polypeptides resulting from autoproteolysis. The electrophoretic position of the molecular mass standards is shown.

Fig. 2. Specific and saturable binding of iodinated DFP-treated cathepsin G to $N$. gonorrhoeae strain FA102. Amount of iodinated cathepsin $\mathrm{G}$ bound specifically, as calculated by total binding minus nonspecific binding; -for details.

Total and nonspecific binding of cathepsin $G$ to whole cells of strain FA102 were distinguished by doing a binding experiment that employed increasing amounts of ${ }^{125} \mathrm{I}$-labelled cathepsin $\mathrm{G}$ in the absence (total binding) or presence (nonspecific binding) of a 50-fold excess of unlabelled cathepsin $\mathrm{G}$. Binding was evaluated after $5 \mathrm{~min}$ incubation at $22^{\circ} \mathrm{C}$ because bactericidal activity of cathepsin $G$ was not expressed under these incubation conditions (Shafer et al., 1986b). Analysis of the calculated specific binding (equal to total minus nonspecific binding) revealed (Fig. 2) that binding was saturable. A binding constant of $10^{8} \mathrm{M}^{-1}$ was calculated when the data were graphed as a Scatchard plot (not shown). 


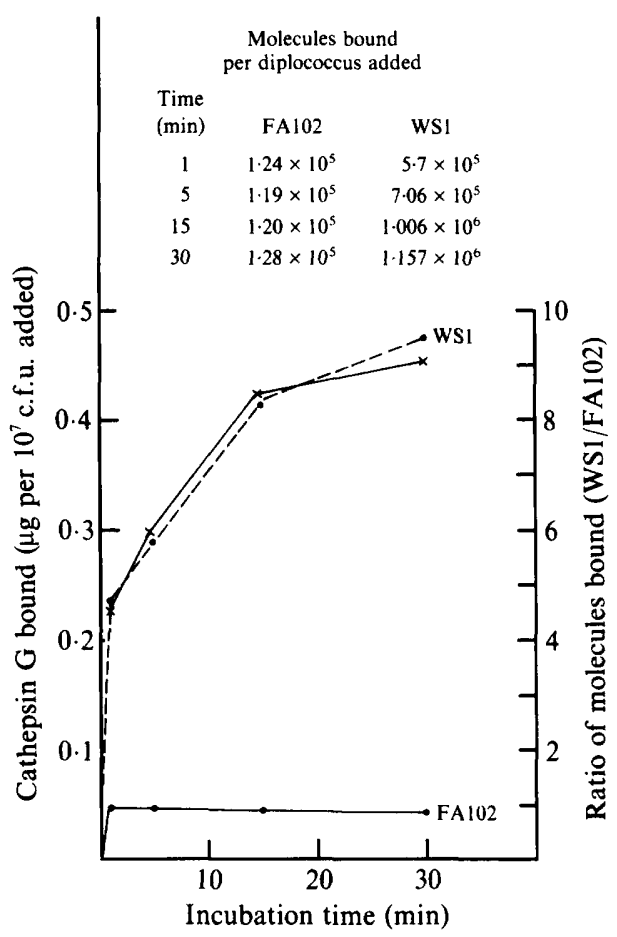

Fig. 3. Loss of core oligosaccharides in LPS due to mutation results in increased total binding of iodinated cathepsin G to gonococci. Isogenic strains FA102 (-—) and WS1 (---) were compared for differences in total binding of cathepsin $\mathrm{G}$ at $22^{\circ} \mathrm{C}$ over a $30 \mathrm{~min}$ incubation period. The calculated number of molecules bound per viable diplococcus added is shown in the insert. $x-\times$, Ratio of number of molecules bound (WS1/FA102).

Table 1. Specific binding of DFP-treated cathepsin G to N. gonorrhoeae FA102 and WS1

Note that the incubation conditions used did not permit bactericidal activity. However, bactericidal action was evident when the incubation temperature was raised to $37^{\circ} \mathrm{C}$ and samples were withdrawn after 15 but not $5 \mathrm{~min}$.

$\begin{array}{lcc}\text { Strain } & \overbrace{\mu \text { ger } 10^{7} \text { c.f.u. added }}^{125 I \text {-labelled cathepsin G bound }} \\ \text { FA102 } & 0.0156 \pm 0.0028(n=3)^{*} & 3.83 \times 10^{4} \\ \text { WS1 } & 0.139 \pm 0.0092(n=3)^{*} & 3.42 \times 10^{5} \\ & \text { * Means } \pm \text { SD, with number of determinations in parentheses. }\end{array}$

Gonococcal LPS structure modulates binding of cathepsin $G$

Isogenic strains FA102 and WS1, differing in LPS subunit molecular mass (Shafer et al., $1986 a$ ) and susceptibility to cathepsin G (Shafer et al., 1986a,b), were compared for their ability to bind DFP-treated cathepsin G. When incubated with $1 \mu \mathrm{g}{ }^{125} \mathrm{I}$-labelled cathepsin $\mathrm{G}$ at $22^{\circ} \mathrm{C}$, strain WS1 exhibited enhanced total binding capabilities (Fig. 3); the number of molecules of cathepsin $\mathrm{G}$ bound per viable diplococcus added for each strain is presented in the table insert. Interestingly, binding of cathepsin G to strain WS1 increased with increasing time of incubation (Fig. 3).

Although the results from the binding experiment described above suggested that the mutation leading to truncation of LPS augmented binding of cathepsin G it did not address 


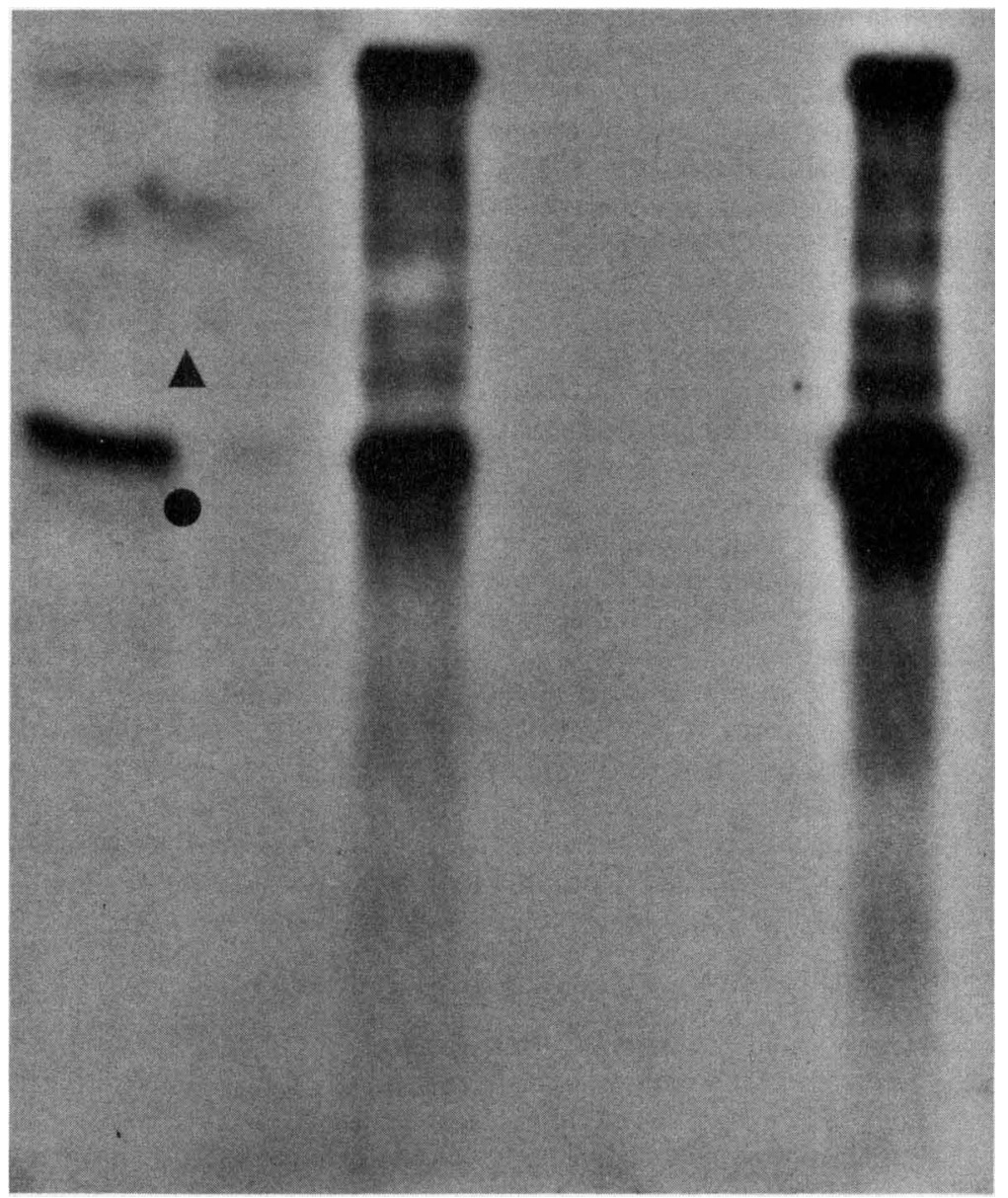

Fig. 4. Detection of iodinated OMPs bound to cathepsin G in immunoprecipitates obtained from SB-12 extracts of strains FA102 and WS1. The surface-iodinated OMP profiles of strains WS1 (lane C) and FA102 (lane F) are shown for reference. The immunoprecipitates obtained from the SB-12 extracts of WS1 treated with cathepsin G (lane A) show a predominant PIA band and faint bands corresponding to PIII (O) and a $45 \mathrm{kDa}$ OMP $(\mathcal{O})$. A much fainter PIA band is visible in the immunoprecipitate from strain WS1 not treated with cathepsin G (lane B). These OMPs were absent in the immunoprecipitates obtained from the SB-12 extracts of parental strain FA102 treated (lane D) or not treated (lane E) with DFP-treated cathepsin G.

whether this was due to increased specific binding. Accordingly, a binding experiment was done using ${ }^{125} \mathrm{I}$-labelled cathepsin $\mathrm{G}$ in the presence and absence of excess unlabelled cathepsin $\mathrm{G}$ incubated with the test strains at $22^{\circ} \mathrm{C}$ for $5 \mathrm{~min}$. Consistent with the known differences in susceptibility to cathepsin G (Shafer et al., 1986a,b), strain WS1 bound about eightfold more molecules of cathepsin G per added c.f.u. than strain FA102 (Table 1).

\section{Enhanced binding of cathepsin $G$ to OMPs due to spontaneous loss of carbohydrates in gonococcal LPS}

Our earlier studies (Shafer \& Morse, 1987) suggested that loss of sugars in the core region of the LPS from strain FA102 augmented the surface exposure of OMPs of strain WS1, thereby increasing their susceptibility to cleavage by cathepsin G. To test whether the OMPs of these strains differed in their ability to bind DFP-treated cathepsin G the strategy used by Joiner $e$ t al. (1983) in their work dealing with binding of C5b-9 to gonococcal OMPs was employed. In brief 
this procedure is centred on the capacity of antiserum against bactericidal substances to coprecipitate ${ }^{125} \mathrm{I}$-labelled OMPs from nonionic detergent extracts of bacteria treated with the appropriate antigen. Surface-iodinated suspensions of strains FA102 and WS1 were incubated in the presence or absence of DFP-treated cathepsin $\mathrm{G}\left(100 \mu \mathrm{g} \mathrm{ml}^{-1}\right)$ at $22^{\circ} \mathrm{C}$ for $30 \mathrm{~min}$. After incubation, the bacteria were collected by centrifugation, washed three times with PBS and treated with $1 \%(\mathrm{w} / \mathrm{v}) \mathrm{SB}-12$ for $30 \mathrm{~min}$ at $37^{\circ} \mathrm{C}$. The debris was pelleted by centrifugation and the extract treated sequentially (see Methods) with goat anti-cathepsin G IgG and rabbit antigoat IgG antiserum. The immunoprecipitates were washed, solubilized and subjected to SDSPAGE. The iodinated gonococcal OMPs were detected by autoradiography of the dried gel. Significantly, the results (Fig. 4, lane A) clearly showed that PIA (serovar PIAl), was the predominant OMP coprecipitated with cathepsin G. Moreover, it was selectively coprecipitated with cathepsin G from SB-12 extracts obtained from suspensions of WS1 treated with cathepsin G. Two additional OMPs (PIII and a $45 \mathrm{kDa}$ polypeptide) were also detected in the immunoprecipitates but in significantly smaller amounts than PIA. The OMP corresponding to PIII migrated identically to authenic PIII that was detected in immunoblots of whole-cell lysates using monoclonal antibody 2E6, known to recognize a surface-exposed epitope of PIII (Tam et al., 1982). The immunoprecipitates from the SB-12 extracts obtained from the controls (absence of added cathepsin G) and cathepsin-G-treated FA102 (lane D) failed to reveal or showed substantially lower amounts of these OMPs (e.g. PIA in lane B but not lanes D and E). The results indicate that sugars present in the LPS of FA102 mask OMPs capable of binding cathepsin G. In control experiments (not shown) these OMPs were not precipitated when the anti-cathepsin $G$ serum was omitted from the reaction mixture. Together these results indicate that sugars in the LPS of FA102 impede the binding of cathepsin G to the OMPs so that the absence of core oligosaccharides in the LPS of WS1 results in greater binding of cathepsin G to OMPs, thus permitting their detection.

\section{DISCUSSION}

The results from this study demonstrate that bactericidal yet enzymically inactive cathepsin $\mathrm{G}$ binds to the surface of gonococci in a saturable manner, indicating that binding is specific. This suggests that binding sites for cathepsin $G$ exist on the surface of the gonococcal outer membrane. The finding that specific antibody against cathepsin $\mathrm{G}$ co-immunoprecipitated at least three OMPs (PIA, PIII and a $45 \mathrm{kDa}$ protein) argues that enzymically inactive cathepsin binds to the surface of gonococci via interactions with OMPs. It remains to be determined whether these OMPs serve as primary binding sites for cathepsin G.

LPS may modulate the susceptibility of gonococci to the bactericidal action of cathepsin $\mathrm{G}$ by sterically reducing the binding of cathepsin $G$ to the gonococcal cell surface. The use of isogenic strains differing in LPS subunit molecular mass has provided two indirect lines of evidence in support of the hypothesis that LPS may mask domains of surface-exposed OMPs that bind cathepsin G. Firstly, OMPs of the LPS mutant (WS1) appeared (Shafer \& Morse, 1987) more susceptible to cleavage in situ by enzymically active cathepsin G than comparable OMPs of the parental strain. Secondly, in the experiments described in this report OMPs PIA1, PIII and a $45 \mathrm{kDa}$ polypeptide co-immunoprecipitated with cathepsin $\mathrm{G}$ and could be detected only with extracts obtained from the mutant WSI. Failure to observe coprecipitation of OMPs, notably PIA, with extracts obtained from the parental strain FA102 was predumably due to the much reduced ability of this strain to bind cathepsin G. It is interesting to note that the $45 \mathrm{kDa}$ OMP had a similar electrophoretic mobility to the recently described (Pohlner et al., 1987) C-terminal portion of the pre-IgA protease which exists as an OMP after autoproteolytic cleavage, but additional work would be required to confirm this identity.

Work by others has shown that LPS associates with OMPs including PI (Hitchcock, 1984) and that PI is in turn complexed with PIII (McDade \& Johnston, 1980; Newhall et al., 1980). Thus, it was not surprising that these OMPs coprecipitated when the SB-12 extracts of cathepsin-G-treated gonococci were treated with antibody directed against cathepsin G. At present it is not clear whether this was due to binding of cathepsin G to LPS, PI or PIII, or a 
combination of these surface components. Mutants lacking or having altered surface domains of these OMPs will help in distinguishing between these possibilities.

Detailing the molecular events leading to the binding of cathepsin $G$ to the surface of gonococci and how this leads to bacterial death is clearly relevant since nonoxidative killing systems may be largely responsible for intraphagosomal death of gonococci. By understanding these events it may be possible to understand why some gonococci have appeared (Casey et al., 1986) to persist within PMNs.

I thank J. Travis for providing the anti-cathepsin G IgG, R. Vogler for procuring granulocyte concentrates, V. Onunka and L. Martin for technical assistance, $K$. Wilkinson for suggestions regarding the binding assays, H. K. Ziegler and D. Stephens for critical review of the manuscript and the encouragement of J. K. Spitznagel. This work was supported by PHS grants AI 21150 (W.M. S.), AI 17662 (J. K. Spitznagel) and AI 22148 (W. Shafer and S. A. Morse) and the Veterans Administration Research Service.

\section{REFERENCES}

BRADFORD, M. (1976). A rapid and sensitive method for the quantitation of microgram quantities of protein utilizing the principle of protein-dye binding. Analytical Biochemistry 72, 248-254.

Casey, S. G., Shafer, W. M. \& Spitznagel, J. K. (1986). Neisseria gonorrhoeae survive intraleukocytic oxygen-independent antimicrobial capacities of anaerobic and aerobic granulocytes in the presence of pyocin lethal for extracellular gonococci. Infection and Immunity 52, 384-389.

Farley, M. F., Shafer, W. M. \& Spitznagel, J. K. (1987). Antimicrobial binding of a radiolabeled cationic neutrophil granule protein. Infection and Immunity 55, 1536-1539.

Hiтchсоск, P. J. (1984). Analyses of gonococcal lipopolysaccharide in whole cell lysates by sodium dodecyl sulfate-polyacrylamide gel electrophoresis: stable association of lipopolysaccharide with the major outer membrane protein (protein I) of Neisseria gonorrhoeae. Infection and Immunity 46, 202212.

Joiner, K. A., Warren, K. A., Brown, E., Swanson, J. \& Frank, M. M. (1983). Studies on the mechanism of bacterial resistance to complement-mediated killing. IV. C5b-9 forms high molecular weight complexes with bacterial outer membrane constituents on serum-resistant but not on serum-sensitive Neisseria gonorrhoeae. Journal of Immunology 131, 1443-1451.

JUDD, R. C. (1982). Surface peptide mapping of protein $I$ and protein III of four strains of Neisseria gonorrhoeae. Infection and Immunity 37, 632-636.

LAEMMLI, U. K. (1970). Cleavage of structural proteins during the assembly of the head of bacteriophage T4. Nature, London 227, 680-685.

McDADE, R. L. \& Johnston, K. H. (1980). Characterization of serologically dominant outer membrane proteins of Neisseria gonorrhoeae. Journal of Bacteriology 141, 1183-1191.

Newhall, W. J., Sawyer, W. D. \& HaAk, R. A. (1980). Crosslinking analysis of the outer membrane proteins of Neisseria gonorrhoeae. Infection and Immunity 28, 785-792.

ODEBERG, H. \& Olsson, I. (1975). Antibacterial activity of cationic proteins from human granulocytes. Journal of Clinical Investigation 56, 1118-1124.

Pohlner, J., Halter, R., Beyreuther, K. \& MEYer, T. F. (1987). Gene structure and extracellular secretion of Neisseria gonorrhoeae $\operatorname{IgA}$ protease. Nature, London 325, 458-462.

Rest, R. F., CoOney, M. H. \& Spitznagel, J. K. (1977). Susceptibility of lipopolysaccharide mutants to the bactericidal action of human neutrophil lysosomal fractions. Infection and Immunity 16, 145151.

REST, R. F., Fischer, S. H., INGHAM, Z. Z. \& JoNES, J. F. (1982). Interactions of Neisseria gonorrhoeae with human neutrophils: effect of serum and gonococcal opacity on phagocytic killing and chemiluminescence. Infection and Immunity 36, 737-742.

ShAFER, W. M. \& MoRSE, S. A. (1987). Cleavage of the protein III and major iron-regulated protein of Neisseria gonorrhoeae by lysosomal cathepsin G. Journal of General Microbiology 133, 155-162.

Shafer, W. M., Martin, L. E. \& Spitznagel, J. K. (1984). Cationic proteins isolated from human neutrophil granulocytes in the presence of diisopropyl fluorophosphate. Infection and Immunity 45, 29-35.

Shafer, W. M., Onunka, V. C. \& Hitchcock, P. J. (1986a). A spontaneous mutant of Neisseria gonorrhoeae with decreased resistance to neutrophil granule proteins. Journal of Infectious Diseases 153, 410-417.

Shafer, W. M., Onunka, V. C. \& Martin, L. E. $(1986 \mathrm{~b})$. Antigonococcal activity of human neutrophil cathepsin G. Infection and Immunity 54, 184 188.

Tam, M. R., Buchanan, T. M., Sandstrom, E. G., Holmes, K. K., KNAPP, J. S., SIADAK, A. W. \& NowINSKI, R. C. (1982). Serological classification of Neisseria gonorrhoeae with monoclonal antibodies. Infection and Immunity 36, 1042-1053.

Travis, T. J., Giles, P. J., Porcelli, I., Reily, C. F., Baugh, R. \& Powers, J. (1980). Human leukocyte elastase and cathepsin $\mathrm{G}$ : structural and functional characteristics. CIBA Foundation Symposium: Excerpta Medica 75, 51-68. 